\title{
Frozen elephant trunk repair of aortic aneurysms: How to reduce the incidence of endoleak and reintervention
}

\author{
Sandhir Kandola, FRCS (Vasc), PhD, ${ }^{a}$ Ahmed Abdulsalam, FRCS (C-Th), MSc, ${ }^{b}$ \\ Mark Field, FRCS, DPhil, ${ }^{\mathrm{b}}$ and Robert K. Fisher, FRCS, MD ${ }^{\mathrm{a}}$
}

\begin{abstract}
Background: Frozen elephant trunk (FET) enables treatment of arch and proximal descending thoracic aorta aneurysms. In treating patients with single-stage FET, the relationship of distal stent size to endoleak and reintervention has remained unexamined.
\end{abstract}

Methods: In this retrospective analysis of 63 cases in which FET was used to repair aneurysms between 2008 and 2019, 36 were intended as single-stage procedures. Effective sizing and sealing of distal stents were analyzed by preoperative and postoperative computed tomography angiography (CTA).

Results: During a mean of $25.8 \pm 5.7$ months of CTA follow-up, 10 of $36(28 \%)$ experienced endoleak, and 3 of $36(8 \%)$ had sac expansion. Ultimately, 5 of $13(38 \%)$ underwent thoracic endovascular aneurysm repair. Patients without endoleak or sac expansion were more likely to have stents with $>10 \%$ oversize and a $>30-\mathrm{mm}$ seal in healthy aorta compared with those experiencing these complications ( 11 of $23 \mathrm{vs}$ o of $13 ; P=.0031)$. Conversely, 11 of 36 patients ( $31 \%$ ) with adequately oversized and sealed stents developed fewer endoleaks compared with those without (o of 11 vs 10 of $14 ; P<.0004$ ). Patients with endoleak or sac expansion had smaller mean distal stent oversize and shorter mean sealing length compared with those without endoleak or sac expansion (2.3 $\pm 3.9 \%$ vs $18 \pm 2.9 \%[P=.0023]$ and $1 \pm 0.7 \mathrm{~mm}$ vs $34 \pm 6 \mathrm{~mm}[P=.0005]$, respectively).

Conclusions: We recommend $>10 \%$ distal stent oversize and $>30-\mathrm{mm}$ sealing length to minimize endoleak and reintervention. Increasing multidisciplinary collaboration with endovascular surgeons will improve distal stent planning. (JTCVS Techniques 2020;3:13-20)

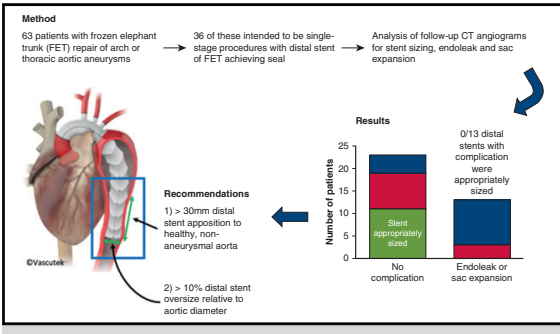

Distal stents with $>10 \%$ oversize and a $>30-\mathrm{mm}$ sealing length do not develop endoleak.

\section{CENTRAL MESSAGE}

We recommend $>10 \%$ distal

stent oversize and $>30-\mathrm{mm}$

sealing length in single-stage

frozen elephant trunk repair of

aortic aneurysmal disease to

prevent endoleak and

reintervention.

\section{PERSPECTIVE}

The frozen elephant trunk enables single-stage treatment of arch and proximal descending thoracic aorta aneurysms, but the relationships of distal stent size to endoleak and need for reintervention remain unexamined. We show that patients with adequately oversized and sealed stents developed fewer endoleaks and required fewer reinterventions compared with those with insufficient oversizing or sealing length to the distal stent.

See Commentaries on pages 21 and 23 .

\footnotetext{
From the a Liverpool Vascular and Endovascular Service, Liverpool University Hospitals NHS Foundation Trust; and ${ }^{\mathrm{b}}$ Department of Cardiothoracic Surgery, Liverpool Heart and Chest Hospital, Liverpool, United Kingdom.

Received for publication May 26, 2020; revisions received May 26, 2020; accepted for publication June 12, 2020; available ahead of print June 20, 2020.

Address for reprints: Sandhir Kandola, FRCS (Vasc), PhD, Liverpool Vascular and Endovascular Service, Liverpool University Hospitals NHS Foundation Trust, Prescot Street, Liverpool UK L7 8XP, United Kingdom (E-mail: s.kandola@uclmail. net).

2666-2507

Copyright (C) 2020 The Authors. Published by Elsevier Inc. on behalf of The American Association for Thoracic Surgery. This is an open access article under the CC BY-NCND license (http://creativecommons.org/licenses/by-nc-nd/4.0/).

https://doi.org/10.1016/j.xjtc.2020.06.015
}

Frozen elephant trunk (FET) repair of the arch and thoracic aorta was introduced as a single-stage solution to extensive aortic arch and proximal thoracic aortic aneurysmal disease. The device consists of a proximal Dacron surgical graft for revascularization of the aortic arch and a distal reinforced stent graft designed to seal in the descending thoracic aorta, connected by a cuff to which the distal arch anastomosis is sutured.

Outcomes from FET procedures appear to compare favorably with those from conventional elephant trunk procedures; a case series from Hannover demonstrated 


\section{Abbreviations and Acronyms \\ CTA = computed tomography angiography \\ DTA $=$ descending thoracic aorta \\ EACTS $=$ European Association for Cardiothoracic Surgery \\ FET $=$ frozen elephant trunk \\ MDT = multidisciplinary team \\ TEVAR $=$ thoracic endovascular aneurysm repair}

decreased in-hospital mortality for FET compared with conventional elephant trunk. ${ }^{1}$ However, reintervention rates to the distal aorta in FET procedures are similar to those in conventional elephant trunk procedures, ${ }^{1}$ which is unexpected given that one of the advantages of the FET device is to avoid further thoracic aortic procedures by creating a primary distal seal. It is also concerning that FET procedures appear to confer an increased risk of spinal cord ischemia compared with conventional surgery. ${ }^{2}$

Endoleak around the distal stent graft may represent a preventable complication resulting in further intervention to prevent sac expansion and rupture; however, the incidence of endoleak is not reported in many retrospective FET case series. ${ }^{3,4}$ Other case series have reported an incidence of endoleak of $11 \%$ to $33 \% .^{5-7}$ However, some endoleaks in these series might have been intentional, in a planned second-stage thoracic endovascular aneurysm repair (TEVAR) to achieve a distal seal. The incidence of endoleak does not vary according to the FET device used. ${ }^{7}$ Rates of endovascular reintervention to the distal aorta, where reported, are between $11 \%$ and $16 \% .{ }^{8,9}$ Overall, the long-term performance of distal stent grafts in FET procedures remains unknown. ${ }^{10}$

In infrarenal aortic aneurysms, an appropriate stent graft oversize is known to prevent endoleak, aneurysm sac expansion, and stent graft migration. ${ }^{11,12}$ However, there are little data on the relationship between sizing of the distal stent and later development of endoleak or ongoing sac expansion in FET procedures. Indeed, the recommendations in the position paper from the vascular domain of the European Association for Cardiothoracic Surgery (EACTS) for FET procedures make no mention of appropriate distal stent sizing. ${ }^{10}$ In the present study, we aimed to examine the relationship between distal stent graft oversize and sealing length and the incidence of endoleak in FET procedures intended to secure a distal seal in the thoracic aorta.

\section{METHODS}

Data were collected retrospectively starting from a database of all FET procedures performed in a single center between 2008 and 2019, containing dates of procedures together with the devices and sizes used. All FET procedures were performed using either the E-Vita Open device (JOTEC, Hechningen, Germany) or the Thoraflex device (Vascutek Terumo, Inchinnan, Scotland). The E-Vita is available in distal stent diameters of 20 to $40 \mathrm{~mm}$ with lengths of 130 to $160 \mathrm{~mm}$, and the diameter of the proximal graft to the aortic arch matches that of the distal stent. The Thoraflex device's distal stent is available in diameters of 24 to $40 \mathrm{~mm}$ and lengths of 100 to $150 \mathrm{~mm}$, with varying combinations of diameters of proximal graft diameter and distal stent diameter. The instructions for the Thoraflex device state that when used for aneurysmal pathology, the distal stent diameter should exceed the thoracic aortic diameter by $15 \%$ to $25 \%$ and the distal stent should seal in $>40 \mathrm{~mm}$ of healthy aorta. The instructions for the E-Vita device do not state sizing recommendations for aneurysmal disease. The choice of device and size was left to the operating cardiac surgeon; however, during the study period, increased contact with endovascular surgeons, particularly for elective cases, might have increased collaboration in the process of device sizing.

Given the lack of consensus about what constitutes an appropriate oversize and distal sealing length, a conservative threshold of $10 \%$ oversize and $30-\mathrm{mm}$ distal seal were set for data analysis. Stents with $<10 \%$ oversize were considered undersized; stents with $<30$-mm contact between stent and healthy landing zone in thoracic aorta were considered inadequately sealed.

Computed tomography angiography (CTA) imaging pre-FET, the first postoperative scan, and all follow-up scans were analyzed for endoleak. All patients underwent annual surveillance CTA. Multiplanar reconstruction in 3 dimensions was used to measure orthogonal aortic diameters and center line reconstruction software was used to measure aortic length. On pre-FET CTA, a healthy landing zone for the distal stent was identified by finding an area of nonaneurysmal (diameter $<40 \mathrm{~mm}$ ) nontortuous aorta with $<2$ mm mural thrombus occupying $<25 \%$ of the aortic circumference. The center lumen line distance of this from the origin of the left subclavian artery was noted. On post-FET CTA, the length of aortic coverage by the distal stent and the diameter of the aorta at this level were measured on the pre-FET scan. This allowed for a comparison between the diameter of the distal stent device and the diameter of the aorta where the distal stent graft landed. The encroachment of the distal stent onto the previously identified appropriate landing zone was measured to calculate the length of the distal seal.

The location of the maximum aortic diameter was used to define the primary location of the aneurysm under treatment (although other parts of the aorta could still have been aneurysmal or ectatic). An endoleak was defined as contrast visible outside the stent graft (in the aneurysm sac or alongside the stent graft) on CTA. Aneurysm sac expansion was defined as an increase in sac size on sequential scans at either the location of the original aneurysm or in the thoracic aorta distal to the stent.

This work was carried out using an existing anonymized prospective database of patients and imaging, collected as part of the organization's normal audit procedures where no individual can be identified retrospectively. Patient consent for inclusion was taken at the time of entry to the database.

Electronic clinic letters from both cardiothoracic and vascular surgery departments were used to identify those patients who underwent further surgery or a non-endoleak distal stent-related complication. Opinions from the treating cardiothoracic surgeons and documentation from multidisciplinary team meetings were used to classify FET procedures as definitive or intended for second-stage TEVAR.

Values are reported as mean \pm SEM or median (range) as appropriate. Comparisons of 2 groups were done using the $t$ test for parametric data and the Mann-Whitney $U$ test for nonparametric data. Comparisons of proportions were performed using the $\chi^{2}$ tests. Statistical significance was considered to have been achieved at a $P$ value $<.05$. 


\section{RESULTS \\ Demographics}

Out of 63 FET procedures performed for aneurysmal disease between 2008 and 2019, 36 were intended as a singlestage procedure, with no preoperative plan for further open or endovascular surgery to the thoracic aorta to achieve aneurysm exclusion. Most of these procedures were performed electively ( 26 of $36 ; 72 \%$ ), with 7 of $36(19 \%)$ performed urgently or on an emergency basis. In 3 cases, the urgency of the FET procedure was unknown.

FET procedures were performed for aneurysmal disease of the ascending aorta ( 10 of $36 ; 27 \%)$, innominate artery ( 2 of $36 ; 6 \%$ ), aortic arch (5 of 36;14\%), left subclavian artery $(11$ of $36 ; 31 \%)$, or descending thoracic aorta (5 of $36 ; 14 \%$ ), as defined by the location of the largest aortic diameter. In 3 cases, the location could not be characterized, owing to incomplete preoperative imaging. The median maximum aortic diameter was $65 \mathrm{~mm}$ (range, 40 to $90 \mathrm{~mm}$ ).

The study cohort comprised 17 males (47\%) and 19 females $(53 \%)$ with a median age of 70 years (range, 33 to 83 years) (Table 1). The FET procedure was performed using the Thoraflex device in 25 cases $(69 \%)$ and with the JOTEC device in 11 cases $(31 \%)$.

TABLE 1. Demographic data of patients undergoing intended singlestage frozen elephant trunk procedure for aneurysmal disease

\begin{tabular}{lc}
\hline \multicolumn{1}{c}{ Characteristic } & Value \\
\hline Number of patients & 36 \\
Age, $\mathrm{y}$, median (range) & $70(33-83)$ \\
Sex, $\mathrm{n}(\%)$ & \\
Male & $17(47)$ \\
Female & $19(53)$ \\
Urgency, $\mathrm{n}(\%)$ & \\
Elective & $26(72)$ \\
Emergency & $7(19)$ \\
Unknown & $3(8)$ \\
Location of primary aneurysm, $\mathrm{n}$ & \\
$\quad(\%)$ & \\
Ascending aorta & $10(27)$ \\
Innominate artery & $2(6)$ \\
Aortic arch & $5(14)$ \\
Left subclavian artery & $11(31)$ \\
Descending thoracic aorta & $5(14)$ \\
Unknown & $3(8)$ \\
Maximum aneurysm diameter, & $65(40-90)$ \\
mm, median (range) & \\
Pathology, $\mathrm{n}(\%)$ & \\
Degenerative & $31(86)$ \\
Pseudoaneurysm & $2(1)$ \\
Connective tissue & $3(1)$ \\
Device, $\mathrm{n}(\%)$ & $25(69)$ \\
Thoraflex & $11(31)$ \\
Jotec & \\
\hline & \\
\hline
\end{tabular}

Patients were followed up with CTA for a mean of $25.8 \pm 5.7$ months after the FET procedure. All patients underwent a postoperative CTA within 4 weeks of the procedure and annual surveillance CTAs thereafter. Patients were followed up in outpatient clinics for a mean of $32 \pm 5.8$ months postprocedure.

\section{Endoleak and Distal Stent-Related Complications}

In 9 of the 36 patients ( $25 \%$ ), an endoleak was visualized on the first postoperative CTA, and 1 further endoleak became evident during CTA follow-up at 9 years postprocedure, making a total of 10 endoleaks $(28 \%)$ postprocedure. All but 1 of the endoleaks were classified as type $1 \mathrm{~b}$ (arising from the distal stent) based on CTA appearance, with 1 type 2 endoleak from a likely intercostal artery (which did not cause sac expansion or require further intervention). In another 3 patients $(8 \%)$, expansion of the original sac or aneurysm dilatation immediately distal to the stent became apparent on follow-up CTA (at 3, 5, and 6 years postprocedure) without any evidence of endoleak before this. Other distal stent-related complications were 1 dissection distal to the stent (3\%) and 1 in-stent thrombosis (3\%) (Figure 1).

Patients with endoleak and those with sac expansion were considered one group, given that the etiology of these complications is likely to be related to the sizing of the distal stent. During follow-up, 5 of 13 patients (38\%) with endoleak or aneurysm sac expansion underwent TEVAR to achieve an aneurysm seal, for an overall reintervention rate of $14 \%$ ( 5 of 36 ). Four TEVAR procedures were performed electively for sac expansion to $>65 \mathrm{~mm}$, and 1 TEVAR procedure was performed urgently (within 48 hours of presentation) due to symptomatic rapid sac expansion. There were no ruptures due to endoleak. TEVAR procedures were performed at a median of 12 months (range, 1 to 120 months) after the FET procedure. At the time of this report, 8 of the patients with endoleak or sac expansion remained in yearly CTA surveillance and were asymptomatic with sac sizes below the $65-\mathrm{mm}$ threshold for consideration of treatment.

\section{Distal Stents with Endoleak Were More Likely to Be Undersized or Undersealed}

Among the 13 patients with endoleak or sac expansion, $10(77 \%)$ had a distal stent $<10 \%$ larger than the aortic diameter where the distal end of the stent ultimately landed (undersized). The remaining 3 patients ( $23 \%$ ) had an appropriately oversized stent but a $<30-\mathrm{mm}$ distal seal in healthy aorta (ie, undersealed). In the 23 patients without endoleak or sac expansion, only $4(17 \%)$ had an undersized distal stent. Patients without endoleak or sac expansion were significantly more likely to have stents that were appropriately oversized and sealed compared with those experiencing these complications (11 of 23 vs 0 of 13 ; $P=.0031$, shaded in green in Figure 2). 


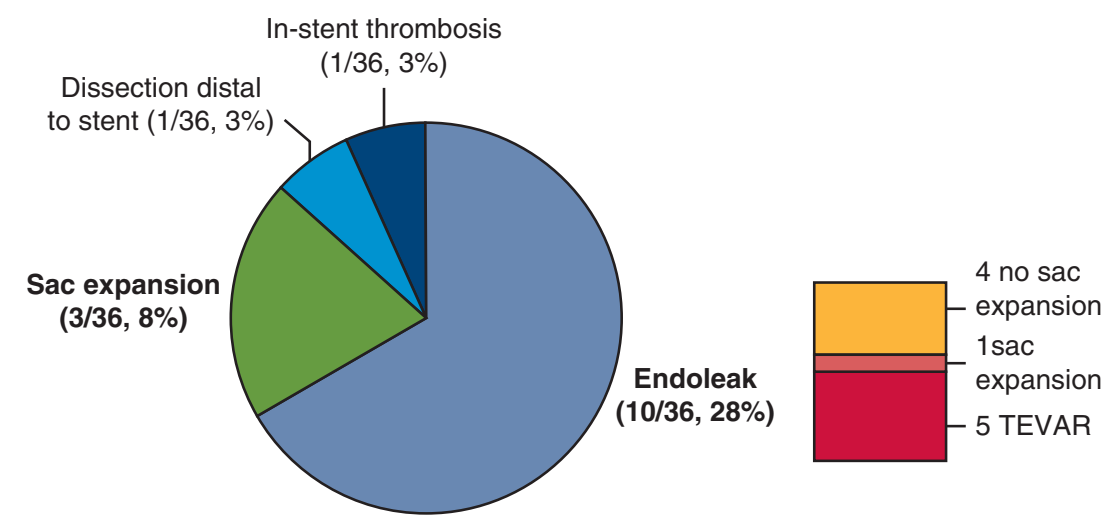

FIGURE 1. Endoleak was the most common distal stent-related complication following intended single-stage frozen elephant trunk (FET) repair of arch and thoracic aortic aneurysms. Distal stent-related complications occurred in 15 of 36 patients (42\%) undergoing FET procedures. Of the 10 patients ( $28 \%$ ) who developed endoleak, 5 underwent thoracic endovascular aneurysm repair (TEVAR) for sac expansion, 1 had sac expansion but with a thoracic aorta diameter below the threshold for treatment, and 4 did not experience sac expansion. Of those developing sac expansion without overt endoleak, none underwent TEVAR, because the diameter of the thoracic aorta remained below the threshold for treatment. This demonstrates that clinically significant endoleak requiring endovascular reintervention remains a problem even in procedures planned as a single-stage FET.

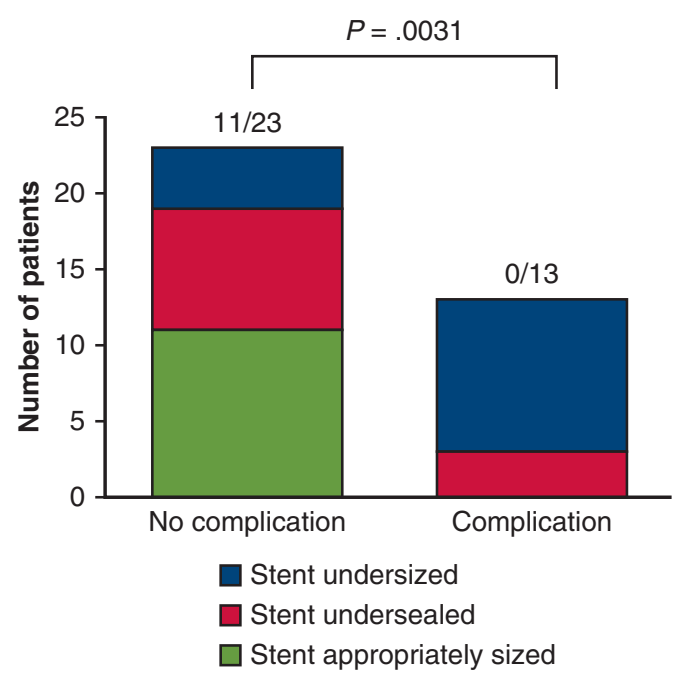

FIGURE 2. Patients with endoleak or sac expansion had undersized and undersealed distal stents. All 13 patients who experienced a sizing-related complication (endoleak or sac expansion; "Complication") had a frozen elephant trunk distal stent that was undersized relative to the aortic diameter where the stent landed (stent diameter $<10 \%$ of aortic diameter, shaded in blue $)$ or inadequately sealed ( $<30 \mathrm{~mm}$ of apposition with healthy, nonaneurysmal aorta; the distal seal, shaded in red). Among the 23 patients who did not experience endoleak or sac expansion ("No complication"), 12 (52\%) had stents that were undersized $(<10 \%$ of aortic diameter) or undersealed $(<30 \mathrm{~mm}$ distal seal), a significantly smaller proportion. Insufficient distal stent oversize in particular resulted in the majority of eventual endoleaks or sac expansions (10 of 13 stents, 77\%). Distal stents not experiencing endoleak or sac expansion were more likely to be appropriately oversized and sealed, compared with those stents experiencing complications (11 of 23 vs 0 of $13 ; P=.0031$ ). This demonstrates the importance of distal stent sizing and planning in reducing sac expansion and endoleak.
Patients with endoleak or sac expansion had significantly smaller mean distal stent oversize compared with patients without endoleak or sac expansion $(2.3 \pm 3.9 \%$ vs $18 \pm 2.9 \% ; P=.0023$ ) (Figure $3, A$ ). Patients with complications had a significantly shorter mean sealing length in healthy aorta than those without complications $(1 \pm 0.7 \mathrm{~mm}$ vs $34 \pm 6 \mathrm{~mm} ; P=.0005)$ (Figure $3, B)$.

\section{Appropriate Distal Stent Graft Sizing and Seal Decreased the Risk of Endoleak}

In 11 of 36 distal stents (31\%), appropriate oversize and sealing length were achieved, whereas 14 of 36 stents $(39 \%)$ were undersized with a too-short distal seal. Appropriately oversized stents developed significantly fewer sizing-related complications compared with undersized and undersealed stents ( 0 of 11 vs 10 of $14 ; P<.0004$ ). Ultimately, 3 of the 14 patients with insufficient oversizing and sealing length $(21 \%)$ underwent TEVAR during the follow-up period (Figures 4 and 5).

In 7 of 14 cases with insufficient oversizing and sealing length, the diameter of the thoracic aorta where the distal stent landed was ectatic at $>36 \mathrm{~mm}$. In these cases, it would not have been possible to achieve $>10 \%$ oversize due to the maximum available distal stent diameter of $40 \mathrm{~mm}$ and length of $160 \mathrm{~mm}$.

\section{DISCUSSION}

Our data on the incidence of endoleak after FET procedures is comparable overall to reports from other small case series $(11 \%-35 \%))^{5-7}$ However, many case series have a longer follow-up period than ours, suggesting that our rate of endoleak may eventually be higher than expected. These patients are all in yearly CTA surveillance. A higher 


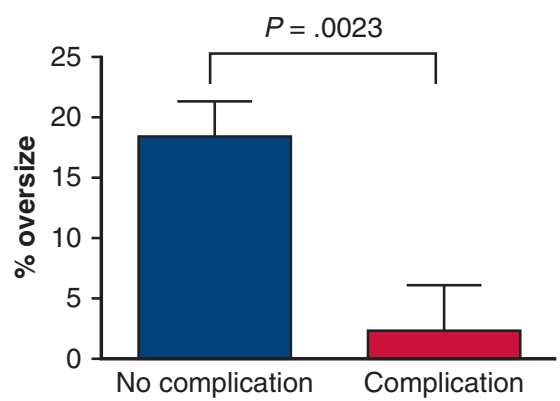

A

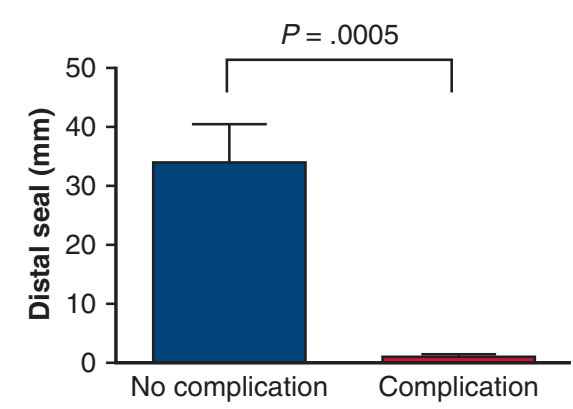

B

FIGURE 3. Frozen elephant trunk distal stents without endoleak or sac expansion had longer distal seal and larger oversize. A, Patients with no distal stentrelated complication (endoleak or sac expansion; "No complication") had a mean aortic diameter oversize of $18 \%$ (above the set threshold of $10 \%$ ) compared with a mean oversize of $2.2 \%$ (below the set threshold of $10 \%$ ) in those with endoleak or sac expansion ("Complication"), demonstrating the importance of distal stent oversize in the prevention of complications. B, Patients without complications ("No complication") had a mean distal seal of $34 \mathrm{~mm}$ (above the set threshold of $30 \mathrm{~mm}$ ) in healthy aorta, compared with $1 \mathrm{~mm}$ (below the set threshold of $30 \mathrm{~mm}$ ) in those with endoleak or sac expansion ("Complication"), demonstrating the importance of an area of apposition of the distal stent against healthy nonaneurysmal aortic wall.

incidence of endoleak in our study could be explained by increased sensitivity for endoleak, given that we retrospectively scrutinized surveillance CTA images specifically for this finding. Post-FET CTA surveillance protocols may also differ, altering the opportunities for detection of endoleak during follow-up.

Our rate of endovascular reintervention to the thoracic aorta after FET (14\%) is comparable to that in the literature, ${ }^{8,9}$ although published case series do not report whether the FET procedure was intended to create a primary seal in the thoracic aorta or the rationale for device sizing. In addition, as the follow-up period increases, future cases of sac expansion will cross the threshold for TEVAR and require future reintervention. It is difficult to compare our series to other endovascular procedures in the thoracic aorta, given that even in TEVAR procedures, the incidence of type $1 \mathrm{~b}$ endoleak has not been quantified owing to a lack of published data. $^{13}$

We have clearly demonstrated that appropriate oversizing and sealing length of the distal stent results in decreased postoperative endoleak and need for reintervention. In some of these cases, preoperative selection of a larger distal stent may have prevented endoleak. In a series of 40 FET procedures, Chu and colleagues ${ }^{14}$ found that selection of an oversized distal stent and a 30- to 40-mm landing zone in the thoracic aorta resulted in no type $1 \mathrm{~b}$ endoleaks (there

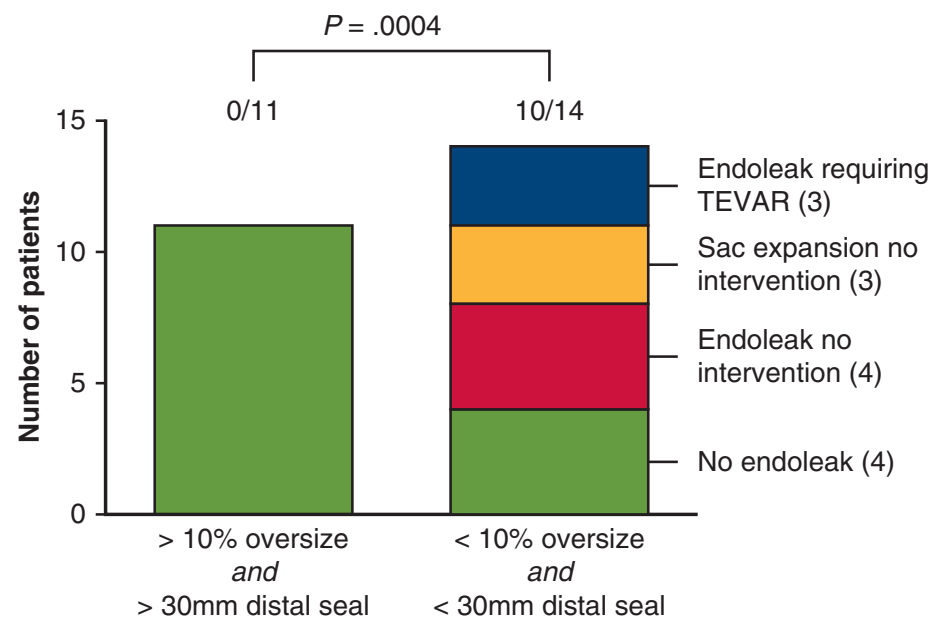

FIGURE 4. Patients with adequate frozen elephant trunk (FET) distal stent sealing size and length did not experience endoleak. In 11 patients with an appropriately oversized distal stent (distal stent diameter $>10 \%$ of aortic diameter) and sufficient distal seal ( $>30$ mm of stent apposition with healthy nonaneurysmal aorta), no endoleak or sac expansion occurred (shaded in green). In the 14 distal stents with neither adequate oversize (distal stent diameter $<10 \%$ of aortic diameter) nor sufficiently long distal seal ( $<30 \mathrm{~mm}$ of stent apposition with healthy nonaneurysmal aorta), 7 developed endoleak ( 3 of which required thoracic endovascular aneurysm repair), and 3 developed sac expansion. Only 4 did not develop a complication. This demonstrates that selection of an appropriately oversized FET distal stent of sufficient length to appose healthy nonaneurysmal aorta is likely to prevent the complications of endoleak and sac expansion, potentially reducing further endovascular reintervention. 

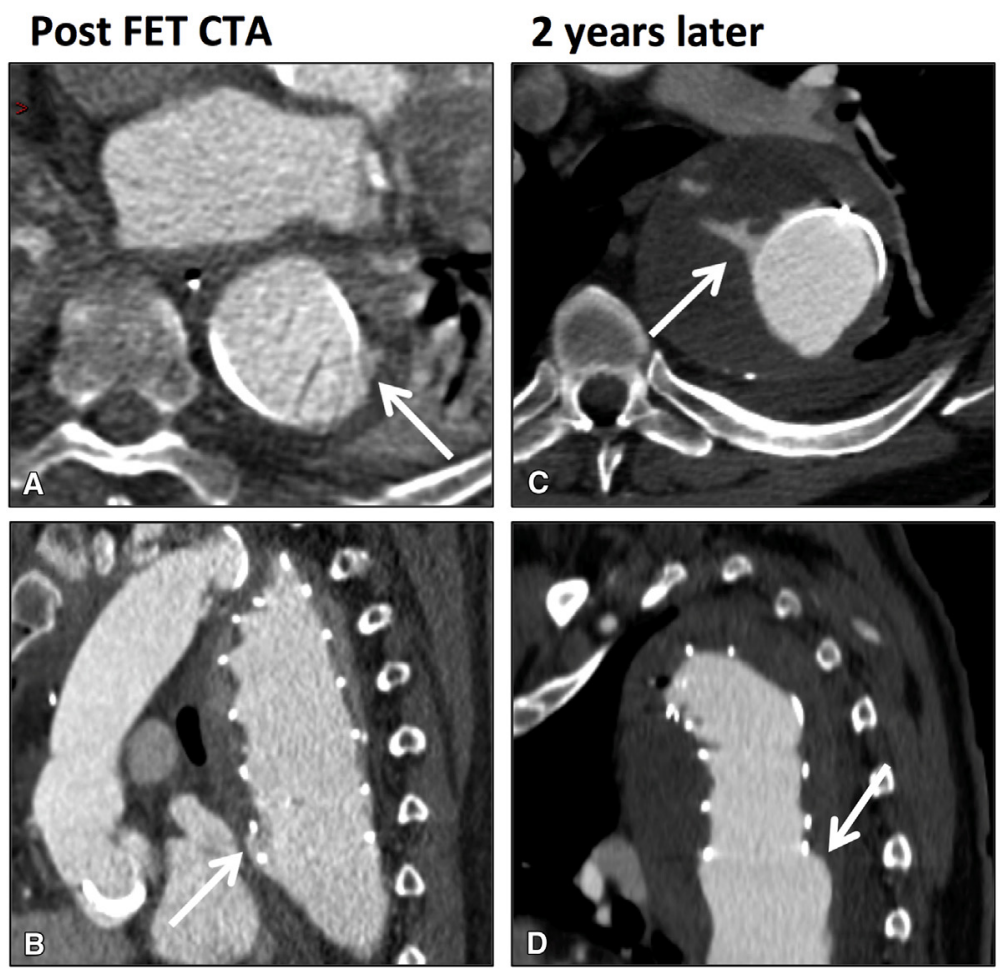

\section{Post TEVAR}

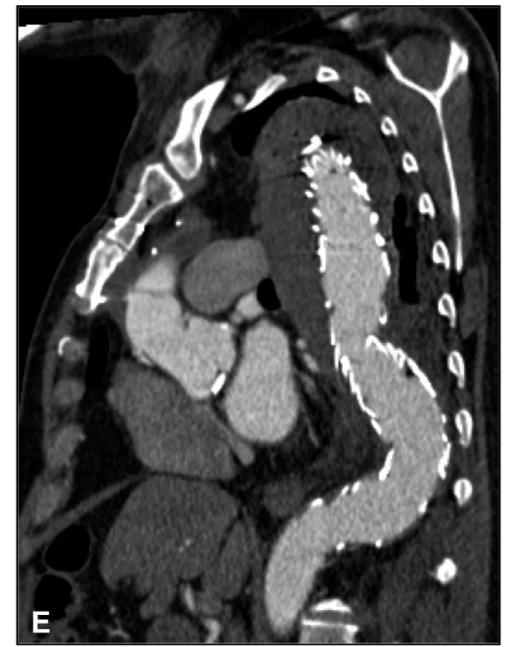

FIGURE 5. Example of late endoleak after intended single-stage frozen elephant trunk ( FET) requiring unplanned thoracic endovascular aneurysm repair (TEVAR) to extend the seal into healthy, nonaneurysmal aorta more distally. A and B, Axial and sagittal computed tomography angiogram (CTA) of distal stent 17 days after a planned single-stage FET procedure, showing small type $1 \mathrm{~b}$ endoleak at the distal aspect of the distal stent (arrows). C and D, Axial and sagittal CTA showing increase in endoleak (arrows) and aneurysm sac expansion 2 years later. E, Post-TEVAR CTA showing extension of distal seal into healthy nonaneurysmal aorta using a further stent-graft and no endoleak. This patient's original FET distal stent was undersized relative to the aortic diameter and had an insufficient sealing length into healthy nonaneurysmal aorta. Preoperative planning of the distal stent length and diameter might have avoided the endoleak and need for reintervention.

were 2 of 40 type 2 endoleaks), suggesting again that preoperative planning can reduce endoleak. Conversely, in Jakob's case series of FET procedures, clinicians selected a $<10 \%$ distal aortic diameter device oversize for the $39 \mathrm{pa}-$ tients with aneurysmal disease, which resulted in a $26 \%$ rate of endovascular reintervention to the thoracic aorta. ${ }^{15}$

In patients requiring TEVAR alone, device manufacturers recommend $10 \%$ to $20 \%$ device oversize compared with aortic diameter and 20 -mm distal sealing length in healthy aorta. Tolenaar and colleagues ${ }^{16}$ found that for TEVAR, a $10 \%$ to $20 \%$ oversize resulted in fewer long term type 1 endoleaks compared with $<10 \%$ or $>20 \%$. Ranney and colleagues ${ }^{17}$ showed that TEVAR performed according to the instructions for use resulted in a $7.3 \%$ reintervention rate over 11 years, lower than that reported for FET procedures over a longer follow-up period. Our thresholds of $10 \%$ oversize and $30-\mathrm{mm}$ distal landing zone are conservative compared with the recommended instructions for use for FET devices, but we have demonstrated that adhering to these criteria resulted in no endoleaks and no reinterventions. Indeed, Damberg and colleagues ${ }^{18}$ recommended a $10 \%$ to $20 \%$ oversize of the distal stent for FET procedures performed for aneurysmal disease. In cases where the thoracic aorta was aneurysmal where the distal stent landed, it might not have been possible to oversize the stent owing to the limited range of stent graft diameters. In these instances, identification of a more distal, nonaneurysmal landing zone and planned extension into this with secondstage TEVAR would have prevented endoleak, with the distal stent of the FET acting as the proximal landing zone for the TEVAR. Greenberg and colleagues ${ }^{19}$ have shown that completion TEVAR in this context is feasible and can be accomplished with minimal mortality. This option needs to be considered before selection of the FET device, given that a significant size discrepancy between the proximal and distal thoracic stent graft diameters is difficult to mitigate using off-the-shelf TEVAR devices.

Moving the landing zone more distally raises concern about the increased risk of spinal cord ischemia due to more extensive coverage of intercostal arteries supplying the thoracic spinal cord, ${ }^{20}$ and in our series this might explain the reluctance to extend aortic coverage with second-stage TEVAR. None of the 36 patients in this series experienced permanent paraplegia or temporary spinal cord 

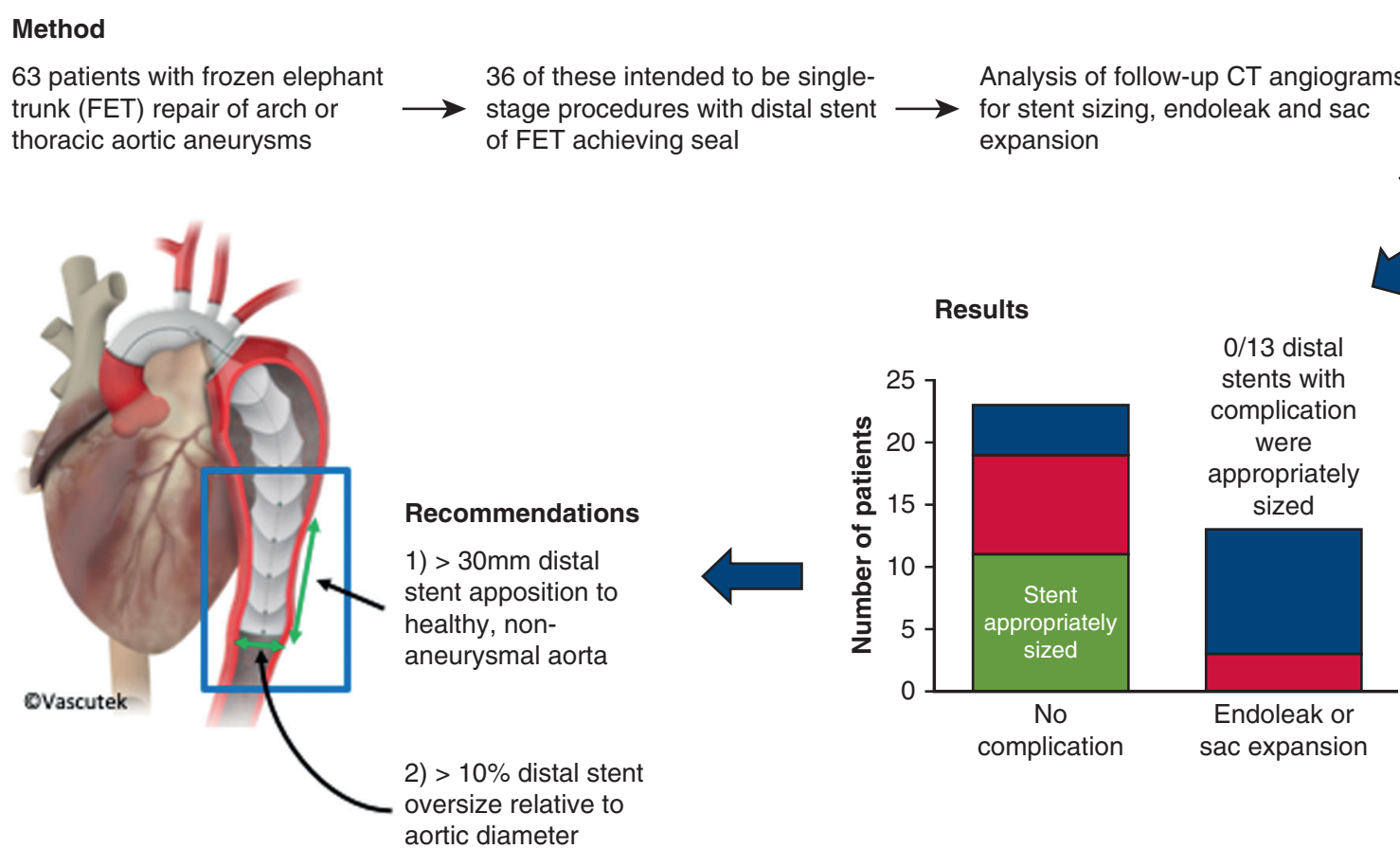

FIGURE 6. Frozen elephant trunk (FET) repair of aortic aneurysms. How to reduce the incidence of endoleak and reintervention. This retrospective study examined 36 FET procedures performed for aortic aneurysmal disease intended as single-stage procedures where the distal stent component of the FET was intended to seal in the thoracic aorta. In 13 of 36 cases (36\%), an endoleak developed or the aneurysm sac continued to expand. All these complications occurred in FETs in which the distal stent was undersized (diameter $<10 \%$ greater than the aortic diameter) or undersealed ( $<30 \mathrm{~mm}$ apposition with healthy aorta distal to the aneurysm). In 5 of $13(38 \%)$ FETs developing endoleak or sac expansion, an unplanned TEVAR was necessary to prevent further sac expansion and rupture. Conversely, no distal FET stents that were appropriately sized developed endoleak or sac expansion and required no further intervention to the thoracic aorta. Thus, we recommend oversizing the distal stent of an FET by $>10 \%$ of the aortic diameter at the proposed landing zone, and to ensure $>30 \mathrm{~mm}$ apposition between the distal stent and healthy nonaneurysmal aorta. Where this is not possible owing to aortic anatomy or availability of stent graft sizes, we would recommend planning a TEVAR extension either concurrently or as a staged procedure. CT, Computed tomography.

injury. Although some have reported that staging these procedures is a protective factor against spinal cord ischemia, ${ }^{21}$ a recent meta-analysis concluded that this was not the case. $^{22}$ The risk of causing paraplegia with TEVAR extension must be balanced against the risks of endoleak, sac expansion, and subsequent rupture.

Involvement of endovascular surgeons in the preoperative planning of these procedures might enable the identification of an appropriate landing zone in the thoracic aorta and facilitate preoperative planning of the distal stent, with or without the need for TEVAR extension. Because the majority of cases are elective, inclusion of endovascular surgeons in multidisciplinary decision making could improve stent graft planning. In emergency cases, the development of aortic emergency rotas could facilitate access to a team member with appropriate expertise in stent graft sizing and planning. The introduction of a multidisciplinary approach has already been shown to contribute to a reduction in operative mortality in the context of acute type A aortic dissection. ${ }^{23}$

This study is limited by the small sample size, which precluded any identification of risk factors for the development of sac expansion or endoleak during CTA follow-up.
Because of the small number of cases and events, our statistical analysis can only be interpreted as descriptive, and inference that these findings apply to larger datasets cannot be computed. The retrospective analysis of data and the short follow-up period limits comparisons with other case series. Stent graft use and planning during the long data collection period might have changed as surgeons learned from previous experience with endoleak.

\section{CONCLUSIONS}

Our results demonstrate the importance of appropriate preoperative sizing of the distal stent in FET procedures. We recommend $>10 \%$ aortic diameter oversize and $>30$ $\mathrm{mm}$ sealing length in healthy aorta, with planning of distal extension with TEVAR if this cannot be achieved with the FET device alone. We encourage an increase in multidisciplinary team collaboration involving endovascular surgeons at an early stage, along with the introduction of all-hours access to an endovascular specialist to facilitate optimal FET planning (Figure 6).

\section{Conflict of Interest Statement}

The authors reported no conflicts of interest. 
The Journal policy requires editors and reviewers to disclose conflicts of interest and to decline handling or reviewing manuscripts for which they may have a conflict of interest. The editors and reviewers of this article have no conflicts of interest.

\section{References}

1. Shrestha M, Beckmann E, Krueger H, Fleissner F, Kaufeld T, Koigeldiyev N, et al. The elephant trunk is freezing: the Hannover experience. J Thorac Cardiovasc Surg. 2015;149:1286-93.

2. Hanif H, Dubois L, Ouzounian M, Peterson MD, El-Hamamsy I, Dagenais F, et al. Aortic arch reconstructive surgery with conventional techniques vs frozen elephant trunk: a systematic review and meta-analysis. Can J Cardiol. 2018;34: 262-73.

3. Leontyev S, Tsagakis K, Pacini D, Di Bartolomeo R, Mohr FW, Weiss G, et al Impact of clinical factors and surgical techniques on early outcome of patients treated with frozen elephant trunk technique by using EVITA open stent-graft: results of a multicentre study. Eur J Cardio-Thorac Surg. 2016;49:660-6.

4. Uchida N, Shibamura H, Katayama A, Sutoh M, Kuraoka M, Ishihara H. Longterm results of the frozen elephant trunk technique for the extensive arteriosclerotic aneurysm. J Thorac Cardiovasc Surg. 2010;139:913-7.

5. Roselli EE, Subramanian S, Sun Z, Idrees J, Nowicki E, Blackstone EH, et al. Endovascular versus open elephant trunk completion for extensive aortic disease. J Thorac Cardiovasc Surg. 2013;146:1408-16; discussion 1416-7.

6. Luehr M, Peters S, Zierer A, Pacini D, Etz CD, Shrestha ML, et al. Aortic events and reoperations after elective arch surgery: incidence, surgical strategies and outcomes. Eur J Cardiothorac Surg. 2018;53:519-24.

7. Ius F, Fleissner F, Pichlmaier M, Karck M, Martens A, Haverich A, et al. Total aortic arch replacement with the frozen elephant trunk technique: 10-year follow-up single-centre experience. Eur J Cardiothorac Surg. 2013;44:949-57.

8. Verhoye JP, Belhaj Soulami R, Fouquet O, Ruggieri VG, Kaladji A, Tomasi J, et al. Elective frozen elephant trunk procedure using the E-Vita Open Plus prosthesis in 94 patients: a multicentre French registry. Eur J Cardiothorac Surg. 2017;52:733-9.

9. Leone A, Beckmann E, Martens A, Di Marco L, Pantaleo A, Reggiani LB, et al Total aortic arch replacement with frozen elephant trunk technique: results from two European institutes. J Thorac Cardiovasc Surg. 2020;159:1201-11.

10. Shrestha M, Bachet J, Bavaria J, Carrel TP, De Paulis R, Di Bartolomeo R, et al. Current status and recommendations for use of the frozen elephant trunk technique: a position paper by the vascular domain of EACTS. Eur J Cardiothorac Surg. 2015;47:759-69.

11. van Prehn J, Schlösser FJV, Muhs BE, Verhagen HJM, Moll FL, van Herwaarden JA. Oversizing of aortic stent grafts for abdominal aneurysm repair: a systematic review of the benefits and risks. Eur J Vasc Endovasc Surg. 2009;38: 42-53.

12. Mohan IV, Laheij RJ, Harris PL, Eurostar Collaborators. Risk factors for endoleak and the evidence for stent-graft oversizing in patients undergoing endovascular aneurysm repair. Eur J Vasc Endovasc Surg. 2001;21:344-9.

13. Belvroy VM, de Beaufort HWL, van Herwaarden JA, Trimarchi S, Moll FL, Bismuth J. Type 1B endoleaks after thoracic endovascular aortic repair are inadequately reported: a systematic review. Ann Vasc Surg. 2020;62:474-83.

14. Chu MWA, Losenno KL, Dubois LA, Jones PM, Ouzounian M, Whitlock R, et al. Early clinical outcomes of hybrid arch frozen elephant trunk repair with the Thoraflex Hybrid graft. Ann Thorac Surg. 2019;107:47-53.

15. Jakob H, Dohle D, Benedik J, Jánosi RA, Schlosser T, Wendt D, et al. Long-term experience with the E-vita Open hybrid graft in complex thoracic aortic disease. Eur J Cardiothorac Surg. 2017;51:329-38.

16. Tolenaar JL, Jonker FHW, Moll FL, van Herwaarden J, Morasch MD, Makaroun MS, et al. Influence of oversizing on outcome in thoracic endovascular aortic repair. J Endovasc Ther. 2013;20:738-49.

17. Ranney DN, Cox ML, Yerokun BA, Benrashid E, McCann RL, Hughes GC Long-term results of endovascular repair for descending thoracic aortic aneurysms. J Vasc Surg. 2018;67:363-8.

18. Damberg A, Schälte G, Autschbach R, Hoffman A. Safety and pitfalls in frozen elephant trunk implantation. Ann Cardiothorac Surg. 2013;2:669-76.

19. Greenberg RK, Haddad F, Svensson L, O'Neill S, Walker E, Lyden SP, et al. Hybrid approaches to thoracic aortic aneurysms: the role of endovascular elephant trunk completion. Circulation. 2005;112:2619-26.

20. Flores J, Kunihara T, Shiiya N, Yoshimoto K, Matsuzaki K, Yasuda K. Extensive deployment of the stented elephant trunk is associated with an increased risk of spinal cord injury. J Thorac Cardiovasc Surg. 2006;131:336-42.

21. Bischoff MS, Brenner RM, Scheumann J, Zoli S, Di Luozzo G, Etz CD, et al. Staged approach for spinal cord protection in hybrid thoracoabdominal aortic aneurysm repair. Ann Cardiothorac Surg. 2012;1:325-8.

22. Preventza O, Liao JL, Olive JK, Simpson K, Critsinelis AC, Price MD, et al. Neurologic complications after the frozen elephant trunk procedure: a metaanalysis of more than 3000 patients. J Thorac Cardiovasc Surg. 2020;160: 20-33.e4.

23. Andersen ND, Ganapathi AM, Hanna JM, Williams JB, Gaca JG, Hughes GC. Outcomes of acute type A dissection repair before and after implementation of a multidisciplinary thoracic aortic surgery program. J Am Coll Cardiol. 2014; 63:1796-803.

Key Words: computed tomography angiography, descending thoracic aorta, frozen elephant trunk, multidisciplinary team, thoracic endovascular aneurysm repair 